
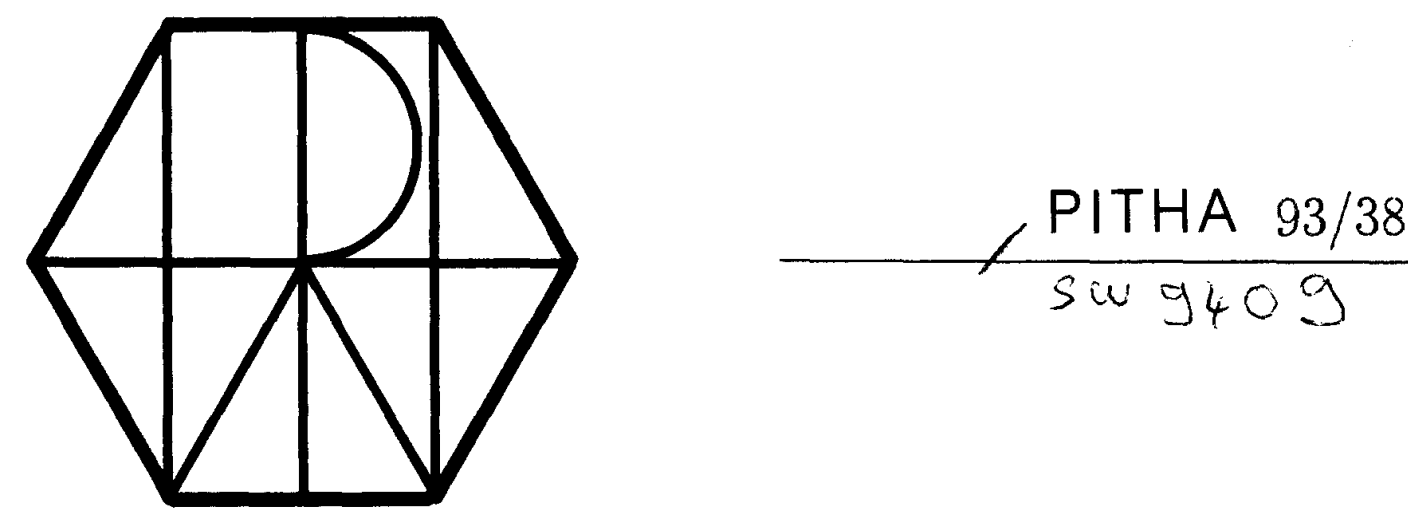

\title{
Calculation of the Electric Field in GaAs Particle Detectors
}

Th. Kubicki, K. Lübelsmeyer, J. Ortmanns

D. Pandouizs, O. Syben, M. Toporowrsky, W. J. Xiao

I. Physikalisches Institut, RWTH Aachen

52056 Aachen, Germany, January 1994

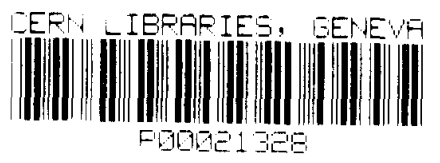

PHYSIKALISCHE INSTITUTE

RWTH AACHEN

Sommerfeldstr.

51 AACHEN, FR GERMANY 


\title{
Calculation of the Electric Field in GaAs Particle Detectors
}

\author{
T. Kubicki, K. Lübelsmeyer, J. Ortmanns, \\ D. Pandoulas, O. Syben, M. Toporowsky, W. J. Xiao
}

I. Physikalisches Institut, RW'TH Aachen

Sommerfeldstraße 28, 52056 Aachen, Germany ${ }^{1}$

\section{Abstract}

We have simulated the electric field in GaAs surface barrier particle detectors in order to explain the observation of reduced charge collection efficiency. Using the Poisson aid continuity equations the electric potential can be calculated numerically in terms of the ionization of deep levels and the spatial variation of the quasi-Fermilevel in GaAs. The dependence of the electric field on different parameters (bias voltage, leakage current, material parameters) has been studied and predictions of the simulation have been experimentally verified.

\footnotetext{
${ }^{1}$ This work was supported by the German Bundesministerium für Forschung und Technologie
} under contract number $056 \mathrm{AC} 11 \mathrm{P}$ 


\section{Introduction}

Experiments at future colliders, like the Large Hadron Collider (LHC), require radiation resistant and fast detectors because of the high luminosity expected $\left(10^{33} \sim 10^{34}\right.$ $\left.\mathrm{cm}^{-2} \mathrm{~s}^{-1}\right)$. Especially for the components near to the beam-line and in the very forward region, doses of $100 \mathrm{Mrad}$ and fluencies of $10^{15}$ neutrons $/ \mathrm{cm}^{2}$ are anticipated within the operation time. The speed and radiation hardness of devices made of GaAs [1] make them very promising candidates for use at future colliders.

We have fabricated and tested several surface barrier diodes made of SI GaAs[2] with two Schottky contacts $(\mathrm{NiCr} / \mathrm{Au})$ as particle detectors [3]. The charge collection efficiency (cce), defined as the ratio of charge measured $(Q)$ to that deposited inside the detector $\left(q_{0}\right)$, has been determined and found not to reach $100 \%$ although the detectors are fully depleted. For irradiation of the Schottky contact with $\alpha$ particles, the cce increases with the applied reverse bias voltage, eventually reaching saturation, but no signal can be measured when the "Ohmic" contact ${ }^{2}$ of a thick detector is irradiated.

The amount of charge $Q$ that can be measured, after a charge $q_{0}$ has been produced at the position $x_{0}$ inside the detector by an incident particle, depends on the mean free drift length of electrons $\lambda_{e}$ and holes $\lambda_{h}$ according to:

$$
\begin{aligned}
Q= & \frac{q_{0}}{d}\left\{\int_{x_{0}}^{d} \exp \left[-\int_{x_{0}}^{x} \frac{d x^{\prime}}{\lambda_{e}\left(\mathcal{E}\left(x^{\prime}\right)\right)}\right] d x+\right. \\
& \left.\int_{0}^{x_{0}} \exp \left[-\int_{x}^{x_{0}} \frac{d x^{\prime}}{\lambda_{h}\left(\mathcal{E}\left(x^{\prime}\right)\right)}\right] d x\right\}
\end{aligned}
$$

where $d$ is the total thickness of the detector with the Schottky contact at $x=0$ and the "Ohmic" contact at $x=d$, and $\mathcal{E}(x)$ is the electric field. The mean free drift lengths $\lambda_{\varepsilon, h}(\mathcal{E}(x))$ are related to the effective lifetimes $\tau_{\varepsilon, h}$ and drift velocities $v_{e, h}(\mathcal{E}(x))$ of the charge carriers according to $\lambda_{e, h}(\mathcal{E}(x))=\tau_{e, h} v_{e, h}(\mathcal{E}(x))$. Fig. 1 shows the dependence of the drift velocity of electrons in GaAs on the electric field. It is proportional to $\mathcal{E}$ up to about $2 \mathrm{kV} / \mathrm{cm}$ with a constant mobility $\mu_{e} \sim 7000$ $\mathrm{cm}^{2} / \mathrm{Vs}[2][8]$, reaches a maximum at about $3.5 \mathrm{kV} / \mathrm{cm}$ and then saturates to about $10^{7} \mathrm{~cm} / \mathrm{s}$.

According to eq. (1), since $\lambda(\mathcal{E})$ is a function of $\mathcal{E}$, the cce depends critically on the electric field distribution in the detector, which for the GaAs detectors is inhomogeneous due to the existence of space charges. To explain the above mentioned phenomena regarding the cce, we constructed a simple model of charge transport by dividing the GaAs detectors into two layers, assuming the life time $\tau$ to be independent of $\mathcal{E}[3]$ :

\footnotetext{
2The "Ohmic" contact mentioned here is actually the forward biased Schottky contact, which behaves like an Ohmic contact.
} 
- An active layer of thickness $X_{A}$, where a strong field $(\mathcal{E}>2 \mathrm{kV} / \mathrm{cm})$ dominates, so that the drift velocity $v$ and the mean free drift lengths $\lambda$ reach high values (in fact they saturate). Consequently the measurable charge $Q$ is high (see eq. (1)).

- A passive layer, where only a weak field exists, so that $\lambda$ is small and little charge is measurable.

It is obvious that due to the existence of a passive layer (values of $\lambda<<d$ ) the cce can not reach $100 \%$. When $\alpha$-particles deposit their energy in this layer, little signal can be observed.

In our model the active layer is located near to the Schottky contact and its thickness $X_{A}$ increases with the applied reverse bias voltage $U$. The cce for $\alpha$ particles incident on the Schottky contact saturates when $X_{A}$ is large enough, since they deposit their total energy in a small region near the surface (distance $<15 \mu \mathrm{m}$ ).

To justify the assumptions made in this model we must calculate the electric field distribution in GaAs detectors and study its dependence on the different parameters (reverse bias voltage $U$, leakage current density $J$, and material parameters).

\section{$3 \quad$ Field Calculation}

The built.in voltage at the Schottky contact and the applied bias voltage cause an electric field in the semiconductor detector, which is modified by the space charges. In an n-type SI GaAs detector the space charges arise from ionized shallow donors, ionized shallow acceptors, ionized deep levels (mainly EL2 [4]) and electrons in the conduction band, whose concentration is denoted here by $N_{D}^{+}, N_{A}^{-}, N_{T}^{+}$and $n$ respectively. The concentration of holes in the valence band is negligible.

We calculate the electric field from the one-dimensional Poisson equation ${ }^{3}$ :

$$
\frac{d^{2} \varphi(x)}{d x^{2}}=-\frac{\rho(x)}{\epsilon}
$$

where $\varphi$ and $\epsilon$ are the electric potential and dielectric constant of the material respectively. The space charge density $\rho(x)$ is:

$$
\rho(x)=e\left[N_{D}^{+}-N_{A}^{-}+N_{T}^{+}(x)-n(x)\right]
$$

At room temperature the shallow donors and acceptors are totally ionized. Thus:

$$
\begin{aligned}
& N_{D}^{+}=N_{D} \\
& N_{A}^{-}=N_{A}
\end{aligned}
$$

\footnotetext{
${ }^{3}$ Since the width and the length of the GaAs detector $\left(5 \times 5 \mathrm{~mm}^{2}\right.$ diode) are both much greater than its thickness $(350 \mu \mathrm{m})$, one can treat it as a one-dimensional problem.
} 
where $N_{D}\left(N_{A}\right)$ is the density of shallow donors (acceptors).

The density of ionized deep levels $N_{T}^{+}$is given in terms of the Fermi-Dirac distribution. Since the thermal equilibrium is disturbed by the current flowing under the influence of the applied voltage, the quasi-Fermi-level has to be used in the FermiDirac function to describe the density of space charges in the semiconductor [5] [6]. The quasi-Fermi-level $E_{F}^{\prime}(x)$ can be described as:

$$
E_{F}^{\prime}(x)=E_{F}-e \chi(x)
$$

where $E_{F}$ is the constant Fermi-level at the Schottky contact and $(-e \chi(x))$ is the position-dependent deviation from it.

Using the quasi-Fermi-level the density of deep level states occupied by electrons is given by:

$$
N_{T}^{\prime}(\varphi(x), \chi(x))=N_{T} \frac{1}{1+\frac{1}{g} \exp \left[\left(E_{T}^{\prime}(x)-E_{F}^{\prime}(x)\right) / k T\right]}
$$

where $E_{T}^{\prime}(x)=E_{T}-\epsilon \varphi(x), N_{T}$ is the density of the deep level states, $E_{T}$ is their energy niveau in the semiconductor in the absence of external influences, and $g$ is the degeneracy factor $[7][8]$.

The density of the ionized deep levels can then be expressed as:

$$
N_{T}^{+}(\varphi(x), \chi(x))=N_{T}-N_{T}^{\prime}(\varphi(x), \chi(x))
$$

Finally, the electron concentration is given by:

$$
\begin{aligned}
n(\varphi(x), \chi(x)) & =N_{C} \exp \left[-\left(E_{C}-e \varphi(x)-E_{F}^{\prime}(x)\right) / k T\right] \\
& =n_{0} \exp [e(\varphi(x)-\chi(x)) / k T]
\end{aligned}
$$

where $N_{C}, E_{C}$ and $n_{0}$ are, respectively, the effective dersity of states in the conduction band, the energy of the conduction band, and the original concentration of electrons in the absence of external influences.

Inserting the charge concentrations from eqs. (4), (6), and (7) into the Poisson eq. (2):

$$
\begin{aligned}
\frac{d^{2} \varphi(x)}{d x^{2}}= & -\frac{e}{\epsilon}\left\{N_{T} \frac{\frac{1}{g} \exp \left[\left(E_{T}-E_{F}-e \varphi(x)+e \chi(x)\right) / k T\right]}{1+\frac{1}{g} \exp \left[\left(E_{T}-E_{F}-e \varphi(x)+e \chi(x)\right) / k T\right]}+\right. \\
& \left.N_{D}^{+}-N_{A}^{-}-n_{0} \exp [(e \varphi(x)-e \chi(x)) / k T]\right\}
\end{aligned}
$$

we obtain a differential equation relating the functions $\varphi(x)$ and $\chi(x)$. 
A second relation between the two functions is given by the continuity equation for electrons, since in $\mathrm{n}$-type GaAs the total current is mainly caused by electrons, their mobility and density being much larger than those of holes.

$$
\frac{d n(x)}{d t}=G-R+\frac{1 d J(x)}{e} \frac{d x}{d x}
$$

where $J, G$ and $R$ are the electron current density, and the generation and recombination rates respectively. In the static case, generation and recombination compensate each other $(G-R=0)$, and the electron concentration does not depend on time, so that $J(x)=$ constant.

The current density $J$ consists of two parts - the drift component $J^{d r}$ and the diffusion component $J^{\text {diff }}$ :

$$
\begin{aligned}
J & =J^{d r}+J^{d i f f} \\
& =-e n v_{e}+e D \frac{d n}{d x}
\end{aligned}
$$

where $D$ is the diffusion coefficient.

Inserting $n$ from eq. (7) in eq. (10) one finds a second differential equation:

$$
\frac{d}{d x} \exp [(e \varphi(x)-e \chi(x)) / k T]=\frac{J+e n_{0} v_{e}(\mathcal{E}(x)) \exp [(e \varphi(x)-e \chi(x)) / k T]}{e D(x) n_{0}}
$$

with $v_{e}(\mathcal{E}(x))$ obtained from the data in fig. 1 and $D(x)$ from the Einstein relation $D(x)=\mu(x) k T / e$, where the mobility $\mu(x)$ is defined as $\mu(x)=v_{\mathcal{e}}(\mathcal{E}(x)) / \mathcal{E}(x)$.

We thus have a system of two differential equations - the Poisson eq. (8) and the current eq. (11), which can be solved for the two unknown functions $\varphi(x)$ and $\chi(x)$ with the following boundary conditions:

$$
\begin{array}{lll}
\varphi(x)=0 & \text { at } x=d & \text { ("Ohmic" contact } \left.{ }^{2}\right) \\
\varphi(x)=U+V_{b i} & \text { at } x=0 & \text { (Schottky contact) } \\
\rho(x)=0 & \text { at } x=d &
\end{array}
$$

Eqs. (12) and (13) represent the conditions resulting from the applied bias voltage $U$ and the built-in diffusion voltage $V_{b i}$. Eq. (14) follows from the fact, that at the "Ohmic" contact $(x=d)$ there is no net space charge. Since in the absence of any external influences $\rho(x)=0$ and $\varphi(x)=\chi(x)=0$ everywhere (neutrality condition), the requirement $\rho(d)=0$ implies that $\chi(d)=0$.

The parameters necessary for the numerical solution of the two coupled differential equations are given in tab. 1 . Their values were chosen to reflect the conditions under which the data used for comparison with the results of our computation were 


\begin{tabular}{|l|l|l|}
\hline \multicolumn{1}{|c|}{ parameters } & values \\
\hline density of deep levels & $N_{T}$ & $5 * 10^{15} \mathrm{~cm}^{-3}$ \\
\hline density of electrons & $n_{0}$ & $10^{7} \mathrm{~cm}^{-3}$ \\
\hline energy of deep levels & $E_{T}$ & $0.825 \mathrm{eV}$ \\
\hline bias Voltage & $U$ & $400 \mathrm{~V}$ \\
\hline leakage current density & $J$ & $20 \mathrm{nA} / \mathrm{mm}^{2}$ \\
\hline temperature & $T$ & $292 \mathrm{~K}$ \\
\hline
\end{tabular}

Table 1: Parameters used for the numerical calculation.

obtained [3].

We consider the Taylor series expansion of the electric potential $\varphi(x)$ at position $x_{s}:$

$$
\varphi\left(x_{s}-\Delta x\right)=\frac{1}{2} \varphi^{\prime \prime}\left(x_{s}\right)(\Delta x)^{2}-\varphi^{\prime}\left(x_{s}\right) \Delta x+\varphi\left(x_{s}\right)
$$

Starting at $x_{s}=d, \varphi(d-\Delta x)$ is calculated from $\varphi(d)$ and $\varphi^{\prime \prime}(d)$ with $\varphi^{\prime}(d)$ as a free parameter. The value of $\varphi(d)$ is given by the boundary condition eq. (12), while $\varphi^{\prime \prime}(d)$ is obtained from the Poisson eq. (2) and boundary condition eq. (14).

For the next iterative step, eq. (15) can now be used again $\left(x_{s}=d-\Delta x\right)$ to compute $\varphi(d-2 \Delta x)$. The derivative $\varphi^{\prime}(d-\Delta x)$ is obtained by differentiating eq. (15) (for $x_{s}=d$ ). The second derivative $\varphi^{\prime \prime}(d-\Delta x)$ is obtained from the Poisson equation. Since the spatial dependence of the charge density $\rho(x)$ is given through the functions $\varphi(x)$ and $\chi(x), \chi(d-\Delta x)$ must first be calculated in order to obtain $\varphi^{\prime \prime}(d-\Delta x)$. The value of the function $\chi(d-\Delta x)$ is obtained from the Taylor series expansion:

$$
\chi(d-\Delta x)=\chi(d)-\chi^{\prime}(d) \Delta x
$$

using the boundary conditions $\varphi(d)=\chi(d)=0$ and eq. (11) to compute $\chi^{\prime}(d)$.

This procedure is repeated till the other end of the detector $(x=0)$ is reached. At this point the calculated value $\varphi(0)$ is compared with the boundary condition eq. (13). The value of the free parameter $\varphi^{\prime}(d)$ is varied and the numerical computation repeated until the boundary condition is satisfied.

\section{Results}

The calculated electric potential in a $350 \mu \mathrm{m}$ GaAs detector at $400 \mathrm{~V}$ reverse bias voltage is shown in fig. $2 \mathrm{a}$. The energy difference $E_{F}^{\prime}-E_{T}^{\prime}$ is shown in fig. $2 \mathrm{~b}$ and the resulting space charge distribution $\rho(x)$ is shown in fig. 2c. Over a region of about 
$300 \mu \mathrm{m}$ starting at, the Schottky contact the difference $E_{F}^{\prime}-E_{T}^{\prime}$ is small $(<0.158$ $\mathrm{eV}$ ). According to eq. (6) a considerable number of deep levels can be ionized there and thus a strong but inhomogeneous electric field exists in this domain. Further away from the Schottky contact, $E_{F}^{\prime}-E_{T}^{\prime}$ is large and few of the deep levels are ionized, resulting in a weak electric field. This field distribution is shown in fig. $2 \mathrm{~d}$. Close to the "Ohmic" contact the electric field is below the value of $2 \mathrm{kV} / \mathrm{cm}$ (passive layer in our model). Toward the Schottky contact, the field increases approximately linearly and the region for which $\mathcal{E}>2 \mathrm{kV} / \mathrm{cm}$ constitutes in our model the active layer of the detector.

The dependence of the thickness of the active layer $\left(X_{A}\right)$ on the applied reverse bias voltage is shown in fig. 3 for a $350 \mu \mathrm{m}$ GaAs detector, assuming a constant leakage current density of $20 \mathrm{nA} / \mathrm{mm}^{2}$. $X_{A}$ increases roughly linearly with the reverse bias voltage.

According to our simulation $X_{A}$ also depends on the leakage current density $J$. The variation of $X_{A}$ with $J$ for a constant bias voltage of $400 \mathrm{~V}$, is plotted in fig. 4 , showing also an approximately linear behaviour.

The extent of the active layer $X_{A}$ depends also on the density $N_{T}$ of deep levels in the detector material. This is shown in fig. 5 for a reverse bias voltage of $400 \mathrm{~V}$ and a leakage current dersity of $20 \mathrm{nA} / \mathrm{mm}^{2}$, where $X_{A}$ is seen to increase strongly with decreasing $N_{T}$ until the active layer spans the whole detector.

We now use our calculation of the electric field distribution, together with the simple model of charge transport [3] outlined previously, to compute the cce for $\alpha$-particles and compare the results with experimental data.

Since $\alpha$-particles penetrating the Schottky contact deposit their total energy in a thin layer in its vicinity, the observed signal is only due to the drifting electrons. The cce $\varepsilon_{Q}^{\alpha}$ for $\alpha$-particles is then given by [3]:

$$
\varepsilon_{Q}^{\alpha}=\frac{1}{d}\left[\int_{0}^{X_{A}} \exp \left(-\frac{x}{\lambda_{e}^{A}}\right) d x+\exp \left(-\frac{X_{A}}{\lambda_{e}^{A}}\right) \int_{X_{A}}^{d} \exp \left(-\frac{x-X_{A}}{\lambda_{e}^{P}}\right) d x\right]
$$

The mean drift length of electrons in the active layer $\lambda_{e}^{A}$ is taken to be constant since the drift velocity of electrons $v_{\epsilon}$ saturates. In the passive layer the mean drift length $\lambda_{e}^{P}$ is small and assumed also to be constant. Under these assumptions eq. (16) follows directly from eq. (1).

In computing $\varepsilon_{Q}^{\alpha}$ the values of $\lambda_{e}^{A}$ and $\lambda_{e}^{P}$ are free parameters, whereas the thickness of the active layer $X_{A}$ is determined by the electric field distribution (through our condition $\mathcal{E}>2 \mathrm{kV} / \mathrm{cm}$ ) and thus, for a given detector, by the applied bias voltage. For different values of the bias voltage fig. 6 shows the cce for $\alpha$-particles calculated with $\lambda_{e}^{A}=175 \mu \mathrm{m}$ and $\lambda_{e}^{P}=50 \mu \mathrm{m}$, the parameter values best describing the experimental data. The latter are also shown in fig. 6 and are seen to agree very 
well with the results of the calculation.

\section{Conclusion}

The electric field distribution in a GaAs surface barrier particle detector has been numerically calculated, using the Poisson and continuity equations and taking into account the density of deep levels in GaAs and the position dependence of the quasiFermi-level.

It is found that in a region close to the "Ohmic" contact the electric field is weak (below $2 \mathrm{kV} / \mathrm{cm}$ ), so that the resulting mean free drift lengths for electrons and holes are small. The field increases approximately linearly toward the Schottky contact, leading to a region with good charge transport properties, which thus defines the capabilities of the diode as a detector. The extent of this "active" region $X_{A}$ is found to increase approximately linearly with the reverse bias voltage applied and with the leakage current density. It also increases strongly with decreasing density of deep levels. The cce for $\alpha$-particles predicted by the computed electric field distribution together with a simple model of charge transport is in very good agreement with measured values.

A numerical calculation of the cce for $\alpha$-particles as well as for minimal ionizing particles considering the dependence $\lambda(\mathcal{E}(x))$ and constant charge carrier lifetimes will be the subject of a future publication.

\section{Acknowledgements}

We would like to thank E. Gerlach and A. Kaser in our Institute for several helpful discussions. We wish also to thank K. Dietz of the Physics Institute, University of Bonn for his careful reading of the manuscript.

\section{References}

[1] C. T. Wang, Introduction to semiconductor technology, GaAs and related compounds, John Wiley \& Sons, (1989), ISBN 0-471-63119-1

[2] American Xtal Technology SI GaAs wafer product

[3] M. Toporowsky et al., Development of GaAs detectors at the RWTH Aachen, presented at the $1^{\text {st }}$ International Conference on Large Scale Applications and Radiation Hardness of Semiconductor Detectors, Florence, Italy, July 7th-9th, 1993 
[4] J. S. Blakemore, Gallium Arsenide, American Institute of Physics, (1987), ISBN $0-88318-525-3$

[5] E. H. Rhoderick, J. Phys. D: Appl. Phys., Vol. 5, (1972), 1920-1929

[6] Q. Y. Ma et al., J. Appl. Phys., Vol. 64, No. 5, (1988)

[7] V. L. Bonč-Bruevič and S. G. Kalašnikov, Halbleiterphysik, Berlin (1982)

[8] S. M. Sze, Physics of semiconductor devices, New York (1983) 


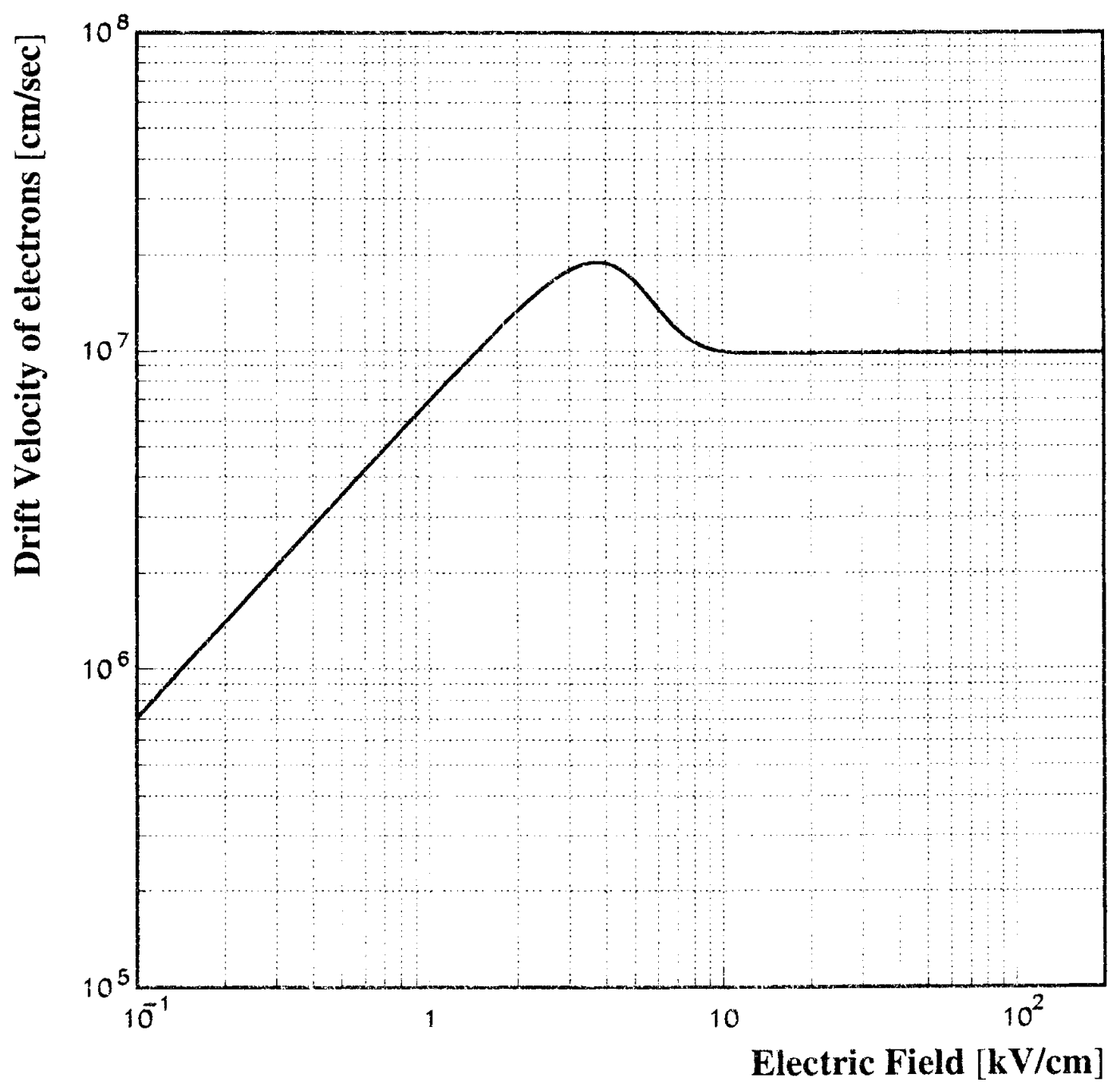

Figure 1: The dependence of the drift velocity of electrons in $\mathrm{GaAs}$ on the electric field. 

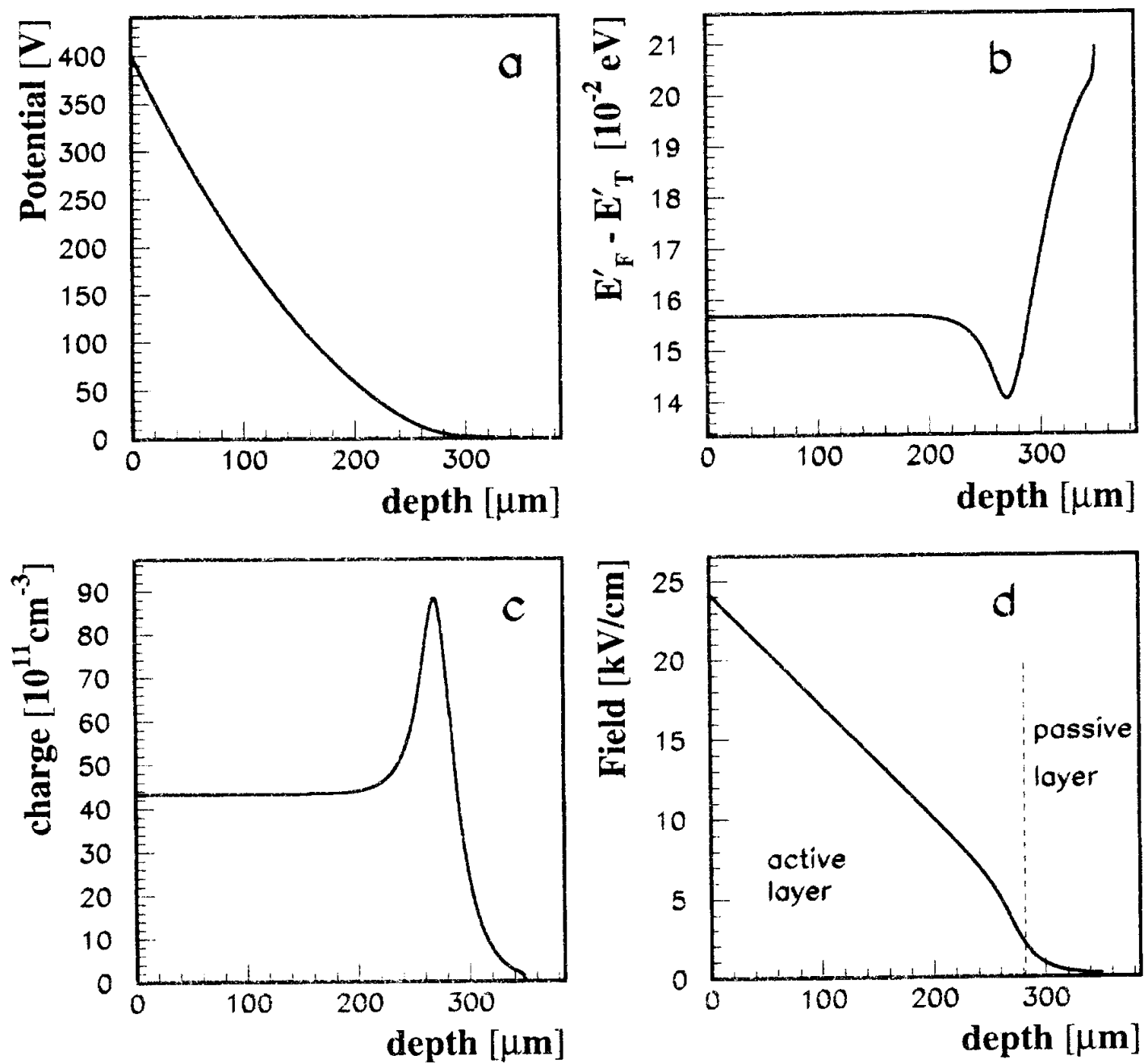

Figure 2: a) Potential-, b) $E_{F}^{\prime}-E_{T}^{\prime}$, c) space charge- and c) electric field distribution in a GaAs detector (thickness: $350 \mu \mathrm{m}$ ). 


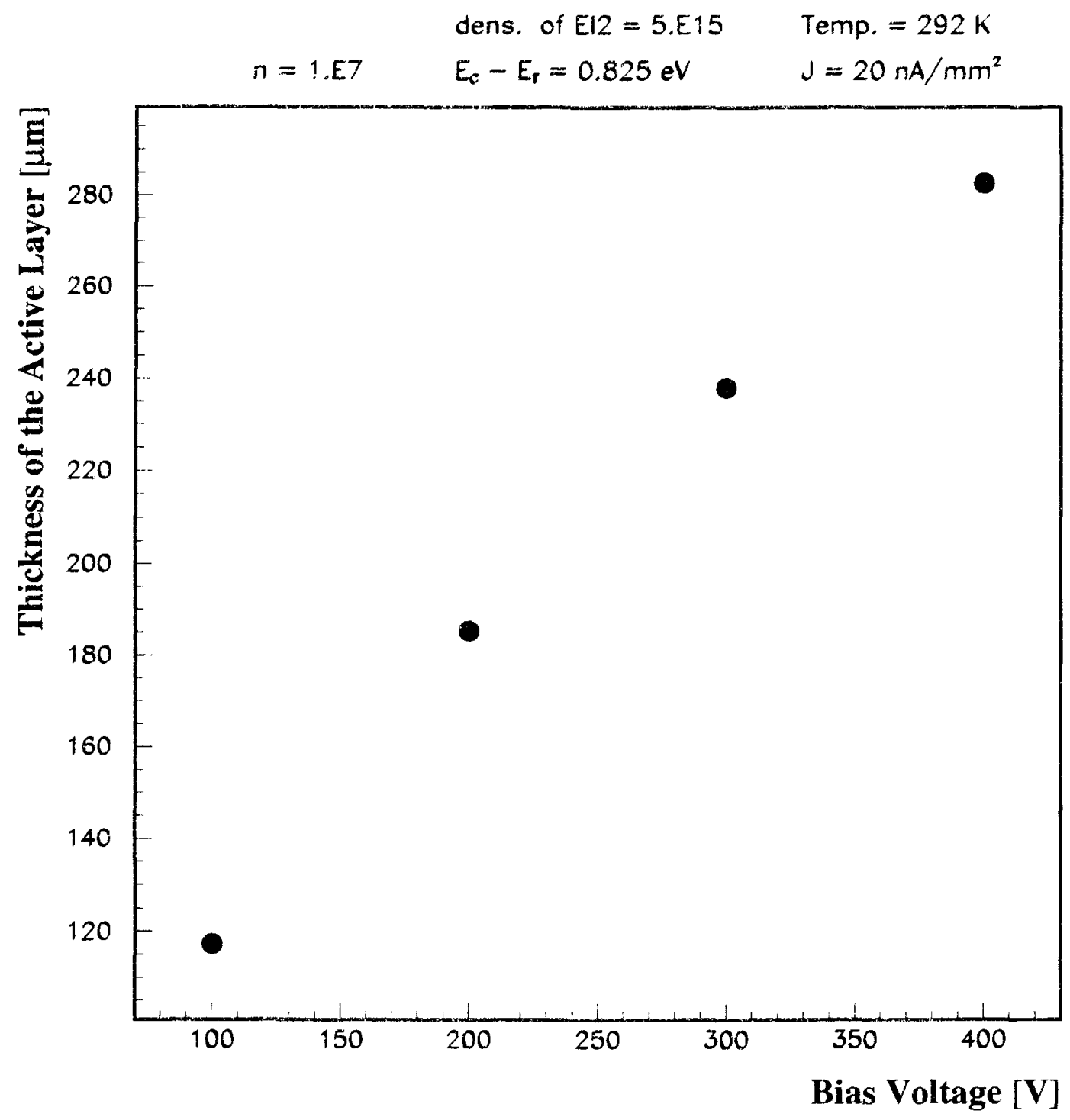

Figure 3: The thickness of the active layer $X_{A}$ as a function of the applied reverse bias voltage in a GaAs detector (thickness: $350 \mu \mathrm{m}$ ). 


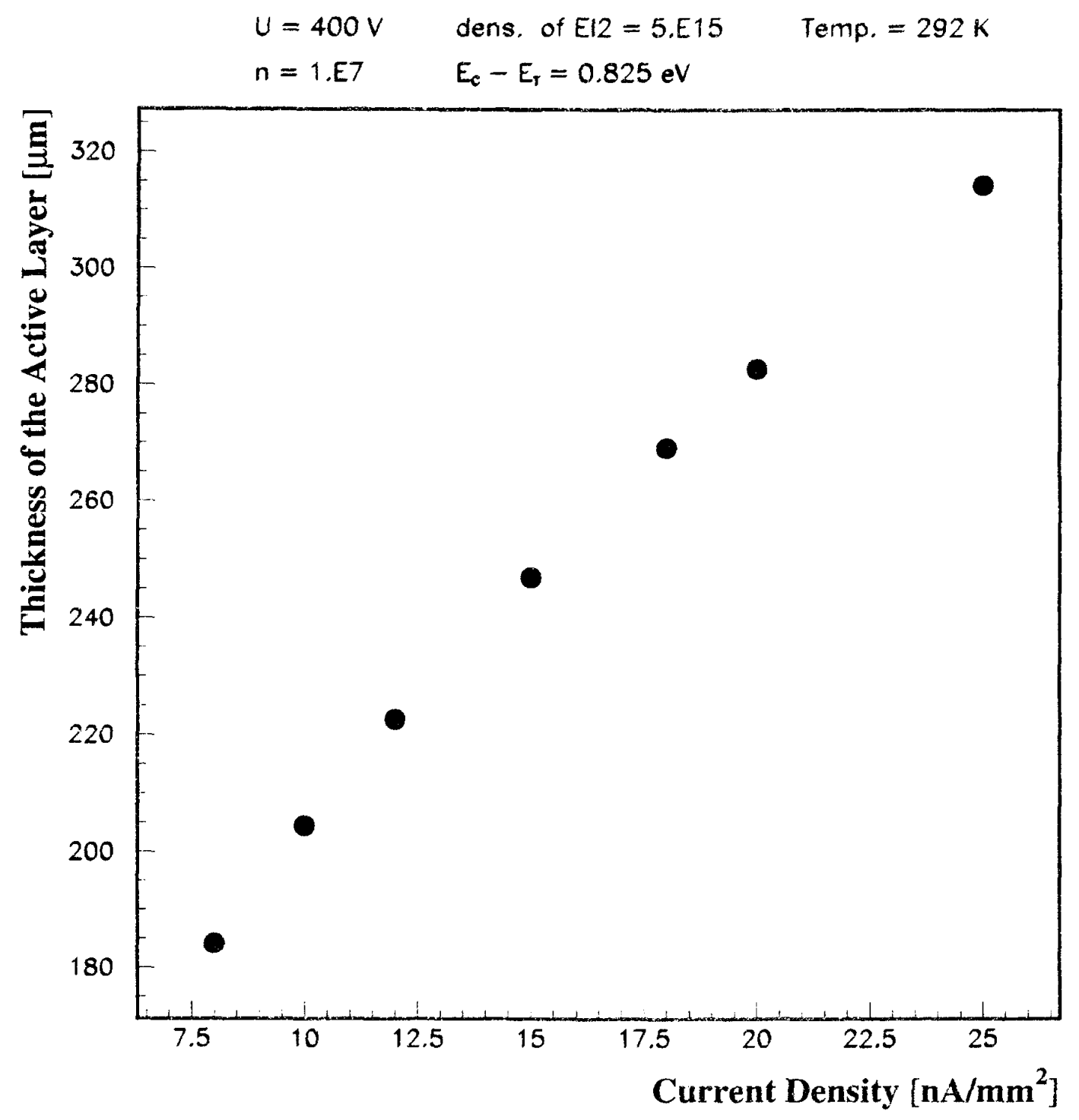

Figure 4: Dependence of the thickness of the active layer $\left(X_{A}\right)$ on the leakage current density for GaAs diodes (thickness: $350 \mu \mathrm{m}$ ) with a Schottky contact. 


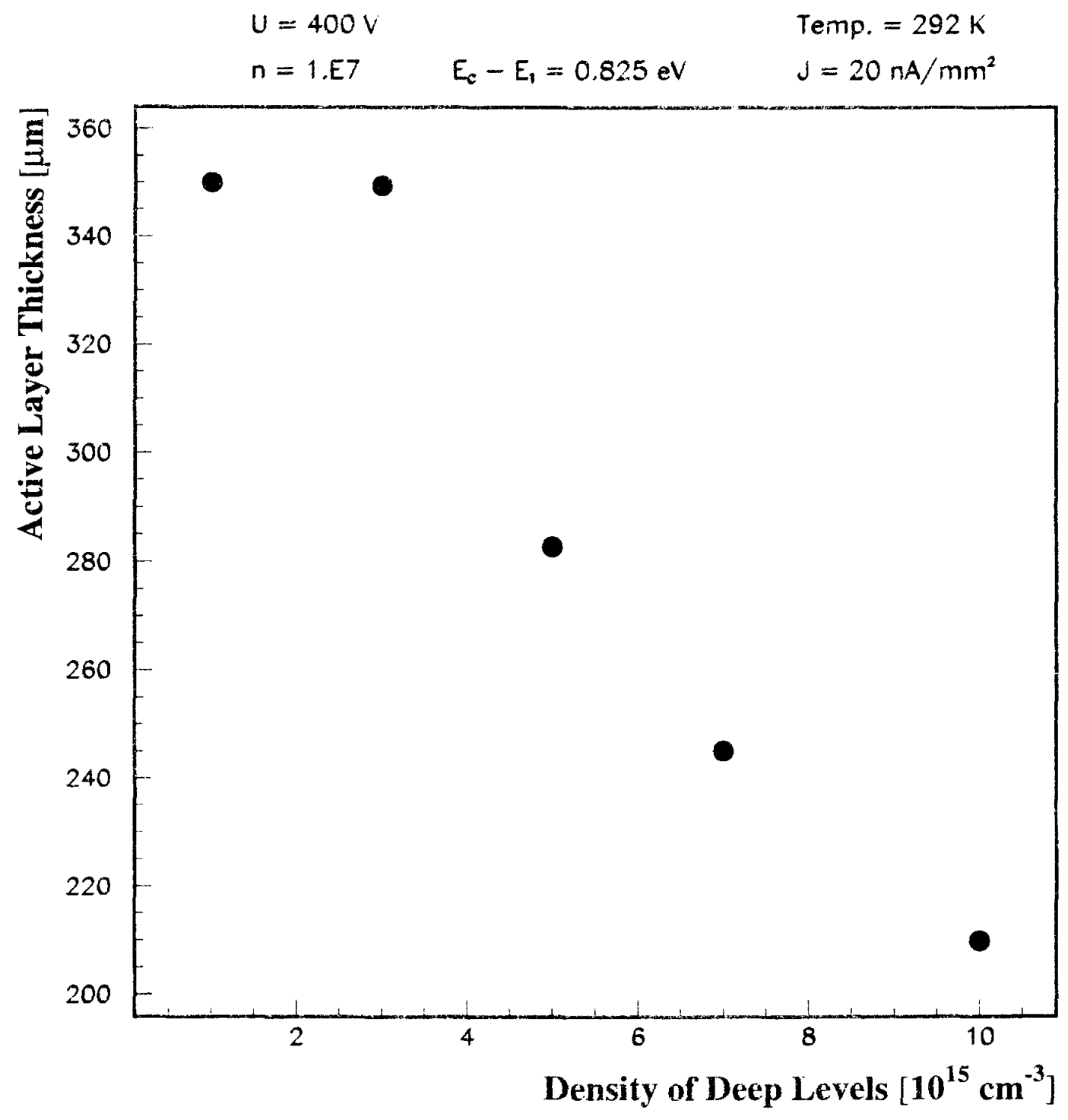

Figure 5: Active layer thickness $X_{A}$ versus the density of the deep levels in a $350 \mu \mathrm{m}$ GaAs detector. 